\title{
VIEWPOINT
}

\section{A "natural experiment" in cardiovascular epidemiology in the early 21 st century}

\author{
A Sekikawa, B Y Horiuchi, D Edmundowicz, H Ueshima, J D Curb, K Sutton-Tyrrell, \\ T Okamura, T Kadowaki, A Kashiwagi, K Mitsunami, K Murata, Y Nakamura, \\ B L Rodriguez, L H Kuller
}

Heart 2003;89:255-257

Despite similar traditional risk factors, morbidity and mortality rates from coronary heart disease in western and non-western cohorts remain substantially different. Careful study of such cohorts may help identify novel risk factors for CHD, and contribute to the formulation of new preventive strategies

T he term "natural experiment" is defined as: "Naturally occurring circumstances in which subsets of the population have different levels of exposure to a supposed causal factor, in a situation resembling an actual experiment where human subjects would be randomly allocated to groups."1 ${ }^{\prime \prime}$ The term is derived from the work of Dr John Snow, who investigated the cholera epidemic which occurred in Broad Street, Golden Square, London in the mid 19th century. He determined the disease transmission by investigating the distribution of cholera cases in relation to contaminated water supplies. ${ }^{2}$

Natural experimentation was first employed in cardiovascular epidemiology in the mid 20th century, sometimes in the form of epidemiological studies with unusual contrast. These studies often give significant insight into prevention of cardiovascular disease. The seven countries study is the first such example. ${ }^{3}$ The study investigated the relation between cardiovascular disease and lifestyle, especially dietary fat intake, across 16 cohorts from seven countries. The mean concentrations of total cholesterol across these cohorts ranged from $4.14-6.73 \mathrm{mmol} / \mathrm{l}$. From the results of the study and others, cholesterol, which we now call a "traditional" or "established" risk factor, has become one of the cornerstones of coronary heart disease (CHD) epidemiology and prevention.

The INTERSALT study investigated the relations between blood pressure and urinary excretion of sodium and potassium across 52 populations in 32 countries. ${ }^{4}$ The mean sodium excretion across populations ranged from $0.2-242 \mathrm{mmol} /$ day. The study revealed that a very low sodium intake was associated with minimal rise of blood pressure with age, and near absence of hypertension. An investigation within one population would not have revealed the finding.

Correspondence to: Dr Akira Sekikawa, 3512 Fifth Avenue, Pittsburgh, PA, USA; akira@pitt.edu

\section{MIGRANT STUDIES}

Another example of a natural experiment is a migrant study. The NI-HON-SAN study is a cross sectional study of cardiovascular disease in migrant Japanese men aged 45-69 years in Hawaii, and California, and Japanese in Japan in the 1960s. ${ }^{5}$ Most of those migrated to the USA in the late 19th or early 20th century, or were second generation Japanese American. By adopting Americanised dietary lifestyle, the concentrations of serum total cholesterol among Japanese American men in the 1960s were higher than that in men in Japan by almost $1.3 \mathrm{mmol} / \mathrm{l}$. The study showed that the CHD mortality was significantly higher in Japanese American men than in men in Japan.

While developed countries have witnessed a dramatic decline in CHD mortality during the 20th century, it remains one of the leading causes of mortality in the western world. Furthermore, a considerable increase in CHD morbidity and mortality is expected in developing countries as their populations age and adopt a more westernised lifestyle. Indeed, prevention of CHD remains one of the major concerns in global health. ${ }^{\circ}$

After the second world war, lifestyle changes in western countries impacted favourably on many CHD risk factors resulting in declines of total cholesterol and blood pressure, and a decrease in the prevalence of cigarette smoking. Conversely, since the second world war, many non-western populations exposed to westernised lifestyles have experienced unfavourable changes in similar CHD risk factors. Despite the resulting similarity in traditional risk factors, however, CHD morbidity and mortality remains substantially different between several post-war birth cohorts in western and non-western countries. Careful study of such cohorts may help identify novel risk factors for CHD and contribute to the formulation of new preventive strategies.

One example is the epidemiological data currently available for post-war birth cohorts that include Japanese natives currently in Japan, Japanese Americans living in Hawaii in the USA, and whites living in the US mainland. Among these cohorts, risk factor profiles for CHD are similar between US white men and Japanese men living in Japan, except for a higher prevalence of cigarette smoking in Japanese men in Japan and a higher prevalence of obesity in the US white men. ${ }^{7}$ The mean concentrations of total cholesterol among men aged 20-34 and 35-44 years in 1988 to 1994 in the USA was $4.82 \mathrm{mmol} / \mathrm{l}$ and $5.34 \mathrm{mmol} / \mathrm{l}$, respectively, and that among 


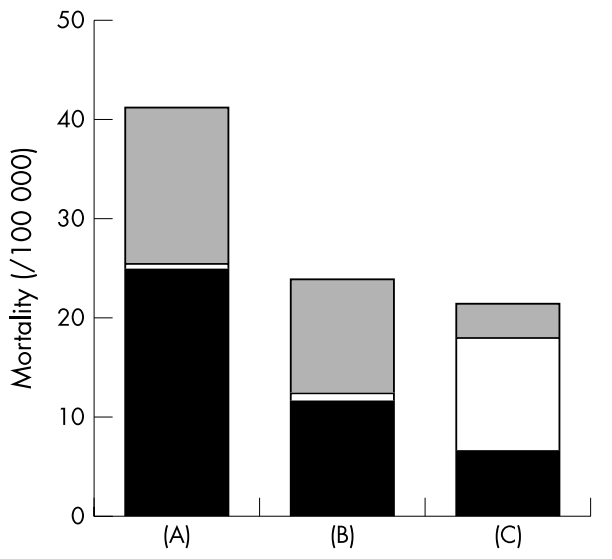

Figure 1 Mortality from coronary heart disease (CHD, black bars), heart failure (white bars), and diseases of the heart other than CHD or heart failure (grey bars) among men aged 35-44. (A) Whites in the USA, (B) Japanese Americans in Hawaii, and (C) Japanese in Japan. Data were average mortality from 1990 to 1998 for whites in the USA and Japanese Americans in Hawaii, and from 1990 to 1997 for Japanese men in Japan. International classification of diseases (ICD) codes (9th revision) for CHD, HF, and diseases of the heart other than CHD and heart failure are 410-414, 428, and $390-429$, respectively. Japan introduced the 10th revision of ICD from 1995, and the corresponding codes were used. Data were from the CDC Wonder for Caucasians in the US, Hawaii Department of Health for Japanese Americans in Hawaii, and from the Ministry of Health and Welfare in Japan for Japanese men in Japan.

men aged 30-39 in Japan in 1989 was $5.21 \mathrm{mmol} / \mathrm{l}$. The mean systolic blood pressure among white men aged 30-39 years in the USA in 1988 to 1991 was $119 \mathrm{~mm} \mathrm{Hg}$, and that among men aged 30-39 in Japan in 1989 was $127 \mathrm{~mm} \mathrm{Hg}$. The mean diastolic blood pressure among white men aged 30-39 years in the USA in 1988 to 1991 was $77 \mathrm{~mm} \mathrm{Hg}$, and that among men aged 30-39 in Japan in 1989 was the same: $77 \mathrm{~mm} \mathrm{Hg}$. The prevalence of cigarette smoking among white men aged 25-34 and 35-44 years in 1990 in the USA was $32 \%$ and $34 \%$, respectively, whereas the prevalence among men aged 30-39 years in Japan in 1989 was $65 \%$. In 1989 , about $15 \%$ of men aged $20-29$, and $20 \%$ of men aged 30-39 in Japan were overweight (body mass index $\geqslant 25.0 \mathrm{~kg} / \mathrm{m}^{2}$ ), whereas in the USA in 1988 to 1994 , the proportion was $47.5 \%$ among men aged $20-34$ and $66.5 \%$ among men aged 35-44 years. Additionally, concentrations of total cholesterol and blood pressure among post-second world war birth cohort in Japanese American men in Hawaii were similar to those among Japanese men in Japan. ${ }^{8}$ Nonetheless, CHD mortality rates in the post-war birth cohort in Japanese men in Japan and Japanese American men in Hawaii remain substantially lower than in US white men (fig l).

Numerous factors can be postulated to explain such a difference in disease rates in these populations. CHD mortality in Japanese men in Japan is likely to be underestimated because of misclassification of death from CHD into heart failure. ${ }^{7}$ Nonetheless, conservatively assuming that $50 \%$ of heart failure cases were caused by CHD, mortality would remain substantially lower than that among US white men. Varying "lag time" between exposure to risk factors and disease occurrence is unlikely to explain the difference. Concentrations of cholesterol in this post-second world war birth cohort of US white men and Japanese men in Japan were very similar in the 1970s. ${ }^{910}$ Stepwise decrement of concentrations of total cholesterol which begins at the age of 12 was observed in both white boys in the USA and boys in Japan, and the mean concentrations of total cholesterol did not differ by
$0.13 \mathrm{mmol} / \mathrm{l}$ between white boys in the USA and boys in Japan in every age from 9 to 15 years where the data are available.

\section{TRADITIONAL RISK FACTORS}

The levels of traditional risk factors are likely to have been similar between Japanese American men in Hawaii and US white men at that time. Very high consumption of omega-3 fatty acids from fish, isoflavonoids from soy products, and alcohol in the Japanese men may account for some of the difference in atherosclerosis formation and CHD morbidity and mortality. ${ }^{112}$ It is also possible that specific genetic polymorphisms differ among these populations and may interact with environmental factors. Reported variation in cholesterol ester transfer protein polymorphisms may affect both high density lipoprotein cholesterol concentrations and atherosclerosis. ${ }^{13}$ Variations in lipoprotein size, distribution, and particle concentration are related to atherosclerosis and CHD, independent of lipid concentrations. ${ }^{14}$ These factors may be effected by genetic polymorphisms of enzymes such as lipoprotein lipase or hepatic lipase and may be influenced by certain environmental factors such as lack of exercise and diets leading to central obesity. ${ }^{15}$

Other opportunities for study in such populations include subclinical atherosclerosis measurements such as coronary calcification detected by electron beam computed tomography, and intimal-medial thickness of the carotid artery measured by ultrasound. ${ }^{16}$ Evaluating and comparing the extent and severity of subclinical atherosclerosis and its relation to various risk factors for CHD might afford the identification of novel risk factors for CHD. Indeed, the careful evaluation of the post-second world war birth cohorts that have adopted traditional western lifestyles may provide new evidence for specific "protective factors", either genetic or environmental, and may be a more powerful epidemiological tool than longitudinal studies in relatively homogeneous populations.

\section{Authors' affiliations}

A Sekikawa, D Edmundowicz, K Sutton-Tyrrell, L H Kuller, University of Pittsburgh, Pittsburgh, USA

B Y Horiuchi, Hawaii Department of Health, Honolulu, USA

H Ueshima, T Okamura, T Kadowaki, A Kashiwagi, K Mitsunami,

K Murata, Y Nakamura, Shiga University of Medical Science, Otsu, Japan

J D Curb, B L Rodriguez, University of Hawaii, Honolulu, USA

\section{REFERENCES}

1 Last JM, ed. A dictionary of epidemiology. Oxford: Oxford University Press, 1995.

2 Snow J. On the mode of communication of cholera. London: J Churchill, 1855.

3 Toshima H, Koga Y, Blackburn H, eds. Lessons for science from the seven countries study. Tokyo: Springer-Verlag, 1994.

4 Rose G, Stamler J, on behalf of the INTERSALT Co-operative Research Group. The INTERSALT study: background, methods and main results. J Hum Hypertens 1989:3:283-8.

5 Worth RM, Kato H, Rhoads GG, et al. Epidemiologic studies for coronary heart disease and stroke in Japanese men living in Japan, Hawaii, and California: mortality. Am J Epidemiol 1975;102:481-90.

6 Beaglehole R. Global cardiovascular disease prevention: time to get serious. Lancet 2001;358:661-3.

7 Sekikawa A, Satoh T, Hayakawa T, et al. Coronary heart disease mortality among men aged 35-44 by prefecture in Japan in 1995-1999 and its comparison with that among white men aged $35-44$ by state in the United States in 1995-1998: vital statistics data in recent birth cohorts. Jpn Circ J 2001;65:887-92.

8 Curb JD, Ueshima H, Nakagawa $\mathrm{H}$, et al. Standardized comparison of CHD risk factor levels in Japanese in Japan and Hawaii: the INTERLIPID study. Presented at the 5th International Conference on Preventive Cardiology. Osaka, Japan.

9 Morrison JA, deGrrot I, Edwards BK, et al. Plasma cholesterol and triglyceride levels in 6,775 school children, aged 6-17. Metabolism 1997;26:1199. 
10 Yano A, Ueshima $\mathrm{H}$, lida $\mathrm{K}$, et al. Primary prevention of cardiovascular disease in youth. In: Komachi Y, eds. Trends in cardiovascular disease. Tokyo: Hoken Dojin-sha, 1987:326-46 [in Japanese].

11 Erdman JW for the AHA Nutrition Committee. Soy protein and cardiovascular disease. A statement for healthcare professionals from the nutrition committee of the AHA Circulation 2000:102:2555-9.

12 Pearson TA. Alcohol and heart disease. Circulation 1996;94:3023-5.

13 Hirano K, Yamashita S, Matsuzawa Y. Pros and cons of inhibiting cholesterol ester transfer protein. Curr Opin Lipid 2000;1 1:589-96.
14 Freedman DS, Otvos JD, Jeyarajah EJ, et al. Relation of lipoprotein subclasses as measured by proton nuclear magnetic resonance spectroscopy to coronary artery disease. Arterioscler Thromb Vasc Biol

15 Hokanson, JE. Functional variants in the lipoprotein lipase gene and risk of cardiovascular disease. Curr Opin Lipid 1999;10:393-9.

16 Greenland P, Abrams J, Aurigemma GP, et al. Prevention conference V. Beyond secondary prevention: identifying the high-risk patient for primary prevention. Noninvasive tests of atherosclerotic burden. Circulation 2000;101:16-22.

\section{IMAGES IN CARDIOLOGY}

Multiple cystic aneurysms in aortitis demonstrated by three dimensional volume rendering images of multislice computed tomography

A 29 year old man presented with slight fever and pulsation at the left neck originating from a gradually expanding palpable mass. Multislice computed tomography (CT) (Aquilion, Toshiba, Tokyo, Japan) was performed with a $1 \mathrm{~mm}$ slice thickness, helical pitch 5.5 , and $100 \mathrm{ml}$ of iodinated contrast material $(300 \mathrm{mg} / \mathrm{ml})$ delivered intravenously at a rate of $3 \mathrm{ml} / \mathrm{s}$. An aneurysm in the left common carotid artery (LCCA) with a mural thrombus was revealed. The aortic arch, proximal portion of the descending aorta (DA) and ascending aorta (AA) appeared to be separated, as if indicating dissection of the lumen. Three dimensional volume rendering images showed collateral arteries around the anterior region of the neck and a cystic lesion from the distal aortic arch to the proximal DA after which the peripheral part of the aorta heads rightward, then downward. In axial source images at this level, the lumen of the distal portion of the
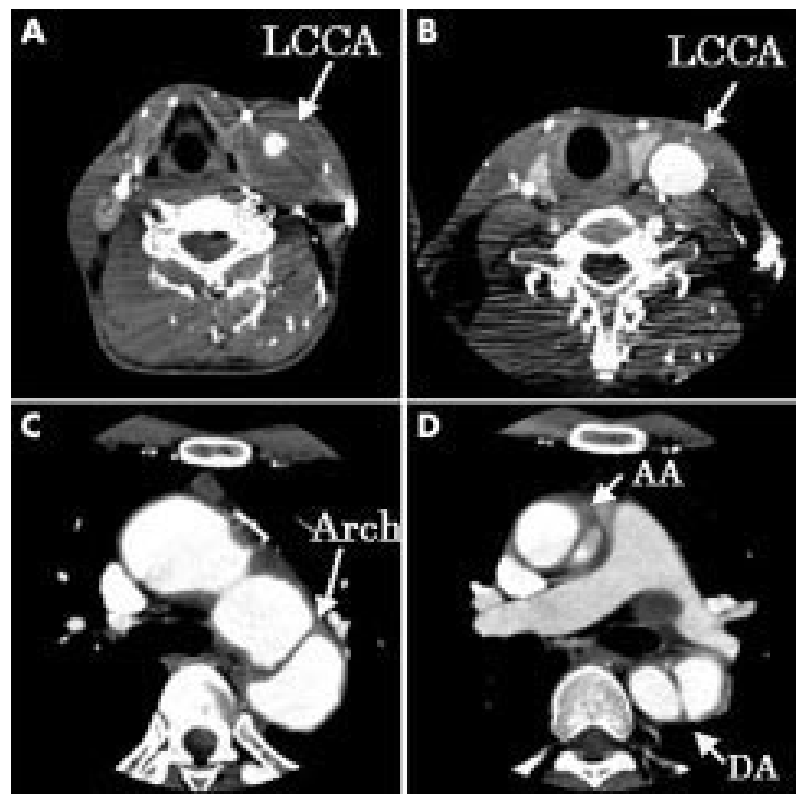

aortic arch and proximal portion of the DA appears separated. Cut plane volume rendering images show distal and proximal portions of the LCCA aneurysm. Stenosis and post-stenotic dilation in the proximal portion of the left subclavian artery (LSA) are observed. Multiple cystic lesions are shown at the inferior border of the aortic arch, which in the axial images (panel D, left) appeared as aortic dissection. Thus, three dimensional volume rendering images showed the presence of multiple cystic aneurysms, but not aortic dissections. CT and blood serum studies indicated inflammation and enabled the diagnosis of aortitis, and steroid therapy was started.

\section{N Funabashi N Komiyama \\ I Komuro komuro-tky@umin.ac.jp}
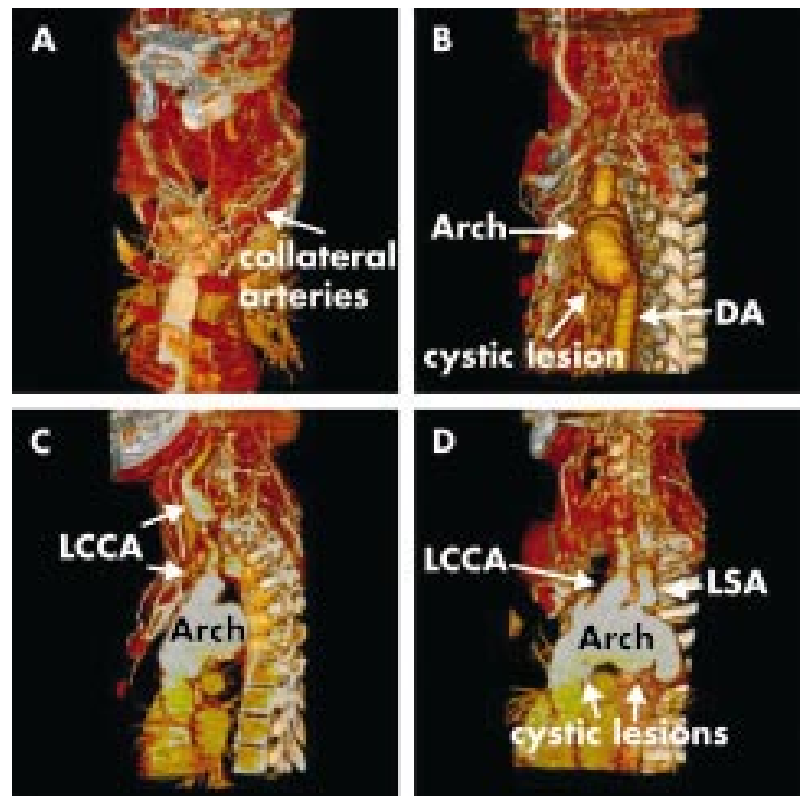